\title{
An Implicit Smooth Conjugate Projection Gradient Algorithm for Optimization with Nonlinear Complementarity Constraints
}

\author{
Cong Zhang1 ${ }^{*}$, Limin Sun ${ }^{1}$, Zhibin Zhu' ${ }^{2}$, Minglei Fang ${ }^{3}$ \\ ${ }^{1}$ Huarui College, Xinyang Normal University, Xinyang, China \\ ${ }^{2}$ School of Mathematics \& Computational Science, Guilin University of Electronic Technology, Guilin, China \\ ${ }^{3}$ College of Science, Anhui University of Science and Technology, Huainan, China \\ Email: ${ }^{*}$ hhcopt@126.com
}

Received 26 July 2015; accepted 15 September 2015; published 18 September 2015

Copyright (C) 2015 by authors and Scientific Research Publishing Inc.

This work is licensed under the Creative Commons Attribution International License (CC BY).

http://creativecommons.org/licenses/by/4.0/

(c) () Open Access

\section{Abstract}

This paper discusses a special class of mathematical programs with equilibrium constraints. At first, by using a generalized complementarity function, the discussed problem is transformed into a family of general nonlinear optimization problems containing additional variable $\mu$. Furthermore, combining the idea of penalty function, an auxiliary problem with inequality constraints is presented. And then, by providing explicit searching direction, we establish a new conjugate projection gradient method for optimization with nonlinear complementarity constraints. Under some suitable conditions, the proposed method is proved to possess global and superlinear convergence rate.

\section{Keywords}

Mathematical Programs with Equilibrium Constraints, Conjugate Projection Gradient, Global Convergence, Superlinear Convergence

\section{Introduction}

Mathematical programs with equilibrium constraints (MPEC) include the bilevel programming problem as its special case and have extensive applications in practical areas such as traffic control, engineering design, and economic modeling. So many scholars are interested in this kind of problems and make great achievements, (see [1]-[10]).

\footnotetext{
${ }^{*}$ Corresponding author.
}

How to cite this paper: Zhang, C., Sun, L.M., Zhu, Z.B. and Fang, M.L. (2015) An Implicit Smooth Conjugate Projection Gradient Algorithm for Optimization with Nonlinear Complementarity Constraints. Applied Mathematics, 6, 1712-1726. 
In this paper, we consider an important subclass of MPEC problem, which is called mathematical program with nonlinear complementarity constraints (MPCC):

$$
\begin{array}{ll}
\min & f(x, y) \\
\text { s.t. } & g(x, y) \leq 0, \\
& 0 \leq G(x, y) \perp y \geq 0
\end{array}
$$

where $f: R^{n+m} \rightarrow R, \quad g=\left(g_{1}, g_{2}, \cdots, g_{p}\right)^{\mathrm{T}}: R^{n+m} \rightarrow R^{P}, \quad G=\left(G_{1}, G_{2}, \cdots, G_{m}\right)^{\mathrm{T}}: R^{n+m} \rightarrow R^{m}$ are all continuously differential functions, $(x, y, w) \in R^{n+m+m}$. $G(x, y) \perp y$ denotes orthogonality of the vectors $y$ and $G(x, y)$, i.e., $y^{\mathrm{T}} G(x, y)=0$.

In order to eliminate the complementary constraints, which can not satisfy the standard constraint qualification [11], we introduce the generalized nonlinear complementary function

$$
\phi(a, b, \mu)=a+b-\sqrt{a^{2}+b^{2}+2 \mu},(a, b, \mu) \in R^{2} \times[0, \infty) .
$$

Obviously, the following practical results about function $\phi$ hold:

- if $\phi(a, b, 0)=0$ and $a \neq b$, then

$$
\begin{aligned}
& D_{a}=\frac{\partial \phi(a, b, 0)}{\partial a} \neq 0, D_{b}=\frac{\partial \phi(a, b, 0)}{\partial b}=0, \text { if } b>0, \\
& D_{a}=\frac{\partial \phi(a, b, 0)}{\partial a}=0, D_{b}=\frac{\partial \phi(a, b, 0)}{\partial b} \neq 0, \text { if } a>0 .
\end{aligned}
$$

$$
\phi(a, b, \mu)=0, \mu \geq 0 \Leftrightarrow a \geq 0, b \geq 0, a b=\mu .
$$

By means of the function $\phi$, problem (1.1) is transformed equivalently into the following standard nonlinear optimization problem

$$
\begin{array}{ll}
\min & f(x, y) \\
\text { s.t. } & g_{j}(x, y) \leq 0, \quad j \in I_{1} \triangleq\{1,2, \cdots, p\}, \\
& c_{j}(x, y, w)=w_{j}-G_{j}(x, y)=0, \quad j \in I_{2} \triangleq\{1,2, \cdots, m\}, \\
& \phi\left(y_{j}, w_{j}, \mu\right)=0, \quad j \in I_{2}, \\
& 1-\mathrm{e}^{\mu}=0 .
\end{array}
$$

Similar to [12], we define the following penalty function

$$
\theta_{c}(x, y, w, \mu)=f(x, y)-c \sum_{j=1}^{m}\left(\phi\left(y_{j}, w_{j}, \mu\right)+c_{j}(x, y, w)\right)+c\left(\mathrm{e}^{\mu}-1\right),
$$

where $c>0$ is a penalty parameter. Therefore, our approach consists of solving an auxiliary inequality constrained problem which is defined by

$$
\begin{array}{cl}
\min & \theta_{c}(x, y, w, \mu) \\
\text { s.t. } & g_{j}(x, y) \leq 0, \quad j \in I_{1}, \\
& c_{j}(x, y, w) \leq 0, \quad j \in I_{2}, \\
& \phi\left(y_{j}, w_{j}, \mu\right) \leq 0, \quad j \in I_{2}, \\
& 1-\mathrm{e}^{\mu} \leq 0 .
\end{array}
$$

\section{Preliminaries and Algorithm}

For the sake of simplicity, we denote 


$$
\begin{aligned}
& z=(x, y, w), s=(x, y), t=(y, w), t_{j}=\left(y_{j}, w_{j}\right), \\
& d z=(d x, d y, d w), d s=(d x, d y), \\
& X_{0}=\left\{z \mid g_{j}(s) \leq 0, j \in I_{1}, 0 \leq G(x, y) \perp y \geq 0\right\}, \\
& X_{1}=\left\{(z, \mu) \mid g_{j}(s) \leq 0, j \in I_{1}, c_{j}(z) \leq 0, j \in I_{2}, \phi\left(t_{j}, \mu\right) \leq 0, j \in I_{2}, 1-\mathrm{e}^{\mu} \leq 0\right\}, \\
& r_{i}=r_{i}(z, \mu)= \begin{cases}g_{j}(x, y), & i=j, j \in I_{1}, \\
c_{j}(x, y, w), & i=j+p, j \in I_{2}, \\
\phi\left(t_{j}, \mu\right), & i=j+p+m, j \in I_{2}, \\
1-\mathrm{e}^{\mu}, & i=p+m+m+1 .\end{cases} \\
& T=\{1,2, \cdots, p+2 m+1\}, \\
& h_{i}=h_{i}(z, \mu)=\nabla r_{i}(z, \mu), H_{i}=H_{i}(z, \mu)=\nabla^{2} r_{i}(z, \mu), i \in T, \\
& I(z, \mu)=\left\{i \in T \mid r_{i}(z, \mu)=0\right\}, J_{0}(z, \mu)=\left\{i \in I_{1} \mid r_{i}(z, \mu)=0\right\} .
\end{aligned}
$$

Throughout this paper, the following basic assumptions are assumed.

$\mathbf{H}$ 2.1. The feasible set of (1.1) is nonempty, i.e., $X_{0} \neq \phi$.

H 2.2. The functions $f, g_{j}, G_{j}\left(j \in I_{2}\right)$ are twice continuously differentiable.

H 2.3. $\forall(z, \mu) \in X_{1}$, the vectors $\left\{h_{i}(z, \mu), i \in J_{0}(z, \mu) \cup\left(T \backslash I_{1}\right)\right\}$ are linearly independent.

The following definition and proposition can be refereed to in [13].

Definition 2.1. Suppose that $z^{*}=\left(x^{*}, y^{*}, w^{*}\right) \in X_{0}$ satisfies the so-called nondegeneracy condition:

$$
\left(y_{j}^{*}, G_{j}\left(x^{*}, y^{*}\right)\right) \neq(0,0), \quad j \in I_{2} .
$$

If there exists multipliers $\left(\lambda^{*}, u^{*}, \gamma^{*}\right) \in R^{p+2 m}$ such that

$$
\begin{aligned}
& \nabla f\left(s^{*}\right)+\nabla g\left(s^{*}\right) \lambda^{*}+\nabla G\left(x^{*}, y^{*}\right) u^{*}+\left(\begin{array}{c}
0_{n \times m} \\
E_{m}
\end{array}\right) \gamma^{*}=0, \\
& 0 \leq-g\left(s^{*}\right) \perp \lambda^{*} \geq 0 ; u_{j}^{*}=0, \text { if } G_{j}\left(s^{*}\right)>0 ; \gamma_{j}^{*}=0, \text { if } y_{j}^{*}>0
\end{aligned}
$$

hold, then $s^{*}$ is said to be a $K-T$ point of (1.1).

Proposition 2.1. Suppose that $z^{*}=\left(x^{*}, y^{*}, w^{*}\right) \in X_{0}$ satisfies the so-called nondegeneracy condition (2.2), then $\left(s^{*}, \lambda^{*}, u^{*}, \gamma^{*}\right)$ is a $K-T$ point of (1.1) if and only if $\left(s^{*}, \lambda^{*}, u^{*}, v^{*}\right)$ satisfies

$$
\begin{aligned}
\left(\begin{array}{c}
\nabla_{x} f\left(s^{*}\right) \\
\nabla_{y} f\left(s^{*}\right) \\
0_{m \times 1}
\end{array}\right)+\left(\begin{array}{c}
\nabla_{x} g\left(s^{*}\right) \\
\nabla_{y} g\left(s^{*}\right) \\
0_{m \times 1}
\end{array}\right) \lambda^{*}+\left(\begin{array}{c}
\nabla_{x} G\left(s^{*}\right) \\
\nabla_{y} G\left(s^{*}\right) \\
-E_{m}
\end{array}\right) u^{*}+\left(\begin{array}{c}
0_{n \times m} \\
W^{*} \\
Y^{*}
\end{array}\right) v^{*}=\left(\begin{array}{c}
0_{n \times 1} \\
0_{m \times 1} \\
0_{m \times 1}
\end{array}\right), \\
0 \leq-g\left(s^{*}\right) \perp \lambda^{*} \geq 0,
\end{aligned}
$$

where

$$
v^{*}=\left(v_{j}^{*}, j \in I_{2}\right) \in R^{m}, v_{j}^{*}= \begin{cases}\gamma_{j}^{*} / w_{j}^{*}, & \text { if } w_{j}^{*}=G_{j}\left(s^{*}\right)>0, \\ u_{j}^{*} / y_{j}^{*}, & \text { if } y_{j}^{*}>0,\end{cases}
$$

$Y^{*}=\operatorname{diag}\left(y_{j}^{*}, j \in I_{2}\right)$ and $W^{*}=\operatorname{diag}\left(w_{j}^{*}=G_{j}\left(s^{*}\right), j \in I_{2}\right)$.

Proposition 2.2. (1) $s$ is a feasible point of (1.1) if and only if $(z, \mu)$ with $w=G(s), \mu=0$ is a feasible point of (1.4).

(2) $s^{*}$ is a $K-T$ point of (1) if and only if $\left(z^{*}, \mu_{*}\right)$ with $w^{*}=G\left(s^{*}\right), \mu_{*}=0$ is a $K-T$ point of (1.4).

Proof. (1) According to the property of function $\phi$, the conclusion follows immediately from (1.3). 
(2) Suppose that $s^{*}$ is a $K-T$ point of (1.1). If set $w^{*}=G\left(s^{*}\right)$ and $\mu_{*}=0$, then, from (1), we see $\left(z^{*}, 0\right)$ is a feasible point of (1.4). While, it follows from proposition 2.1 that there exists vector $\left(\lambda^{*}, u^{*}, v^{*}\right)$ such that (2.5) and (2.6) hold. Define

$$
\hat{v}^{*}=\left(\hat{v}_{j}^{*}, j \in I_{2}\right) \in R^{m}, \hat{v}^{*}=\left\{\begin{array}{ll}
y_{j}^{*} v_{j}^{*} / D_{a}\left(t_{j}^{*}\right), & \text { if } y_{j}^{*}>0, \\
w_{j}^{*} v_{j}^{*} / D_{b}\left(t_{j}^{*}\right), & \text { if } y_{j}^{*}=0,
\end{array} i^{*}=-\sum_{j=1}^{m} \frac{\partial \phi\left(t_{j}^{*}, \mu_{*}\right)}{\partial \mu} \hat{v}_{j}^{*} .\right.
$$

So, it is not difficult to prove that $\left(z^{*}, \lambda^{*}, u^{*}, \hat{v}^{*}, l^{*}\right)$ satisfies the $K-T$ system of (1.4), according to (1.3), (2.5) and (2.6).

Conversely, if $\left(z^{*}, \mu_{*}\right)$ is a $K-T$ point of (1.4), then it follows that

$$
w^{*}=G\left(s^{*}\right), \mu_{*}=0 \text { and } \phi\left(t_{j}^{*}, 0\right)=\phi\left(t_{j}^{*}\right)=0,
$$

which shows that $s^{*}$ is a feasible point of (1.1). Suppose $\left(\lambda^{*}, u^{*}, \hat{v}^{*}, l^{*}\right) \in R^{p+2 m+1}$ is a $K-T$ multiplier corresponding to $\left(z^{*}, \mu_{*}\right)$ of (1.4). Define $v^{*}$ as follows:

$$
v^{*}=\left(v_{j}^{*}, j \in I_{2}\right) \in R^{m}, v_{j}^{*}= \begin{cases}D_{b}\left(t_{j}^{*}\right) \hat{v}_{j}^{*} / y_{j}^{*}, & \text { if } y_{j}^{*}>0 ; \\ D_{a}\left(t_{j}^{*}\right) \hat{v}_{j}^{*} / w_{j}^{*}, & \text { if } y_{j}^{*}=0 .\end{cases}
$$

Then, it is easy to see, from (1.2) and the $K-T$ system of (4) at $\left(z^{*}, \mu_{*}\right)$, that $s^{*}$ with the multiplier $\left(\lambda^{*}, u^{*}, v^{*}\right)$ satisfies (2.5) and (2.6). Therefore, we assert $s^{*}$ is a $K-T$ point of (1.1) according to proposition 2.1.

Now, we present the definition of multiplier function associated with $\epsilon$-active set [14].

Definition 2.2. A continuous function $\rho(z, \mu): R^{n+2 m+1} \rightarrow R^{p+2 m+1}$ is said to a multiplier function, if $\left(z^{*}, \mu^{*}\right)$ satisfies the $K-T$ system of (1.5) with corresponding multipliers $\rho\left(z^{*}, \mu^{*}\right)$.

Firstly, for a given point $\left(z^{k}, \mu_{k}\right)$, by using the pivoting operation, we obtain an approximate active $J_{k}=J\left(z^{k}, \mu_{k}\right)$.

Algorithm A:

Step 1. For the current point $\left(z^{k}, \mu_{k}\right) \in X_{1}$ and parameter $\varrho\left(z^{k}, \mu_{k}\right)=\left(\varrho_{i}\left(z^{k}, \mu_{k}\right), i \in T\right) \in R^{p+2 m+1}$. Set $l=0$, $\epsilon_{l}\left(z^{k}, \mu_{k}\right)=\epsilon_{0}$

Step 2. If $\operatorname{det}\left(A_{l}\left(z^{k}, \mu_{k}\right)^{\mathrm{T}} A_{l}\left(z^{k}, \mu_{k}\right)\right) \geq \epsilon_{k, l}$, let $J_{k}=J_{k, l}, A_{k}=A_{l}\left(z^{k}, \mu_{k}\right), l\left(z^{k}, \mu_{k}\right)=l$, stop; otherwise, goto Step 3, where

$$
\begin{aligned}
& J_{k, l}\left(z^{k}, \mu_{k}\right)=\left\{i \in I_{1}\left|-\epsilon_{k, l}\right| \varrho_{i}\left(z^{k}, \mu_{k}\right) \mid \leq r_{i}\left(z^{k}, \mu_{k}\right) \leq 0\right\}, \\
& A_{k, l}=\left\{h_{i}\left(z^{k}, \mu_{k}\right), i \in J_{k} \cup\left(T \backslash I_{1}\right)\right\} .
\end{aligned}
$$

Step 3. $l=l+1, \quad \epsilon_{k, l}=\frac{1}{2} \epsilon_{k, l-1}$, go back to Step 2 .

Lemma 2.1. For any iteration index $k$, algorithm $A$ terminates in finite iteration.

For the current point $\left(z^{k}, \mu_{k}\right)$ and $\epsilon$-active set $J_{k}$, compute

$$
F\left(z^{k}, \mu_{k}\right)=\left(r_{i}\left(z^{k}, \mu_{k}\right), i \in L_{k}=J_{k} \cup\left(T \backslash I_{1}\right)\right), A_{k}=A\left(z^{k}, \mu_{k}\right)=\left(h_{i}\left(z^{k}, \mu_{k}\right), i \in L_{k}\right) .
$$

Now we give some notations and the explicit search direction in this paper.

$$
\begin{aligned}
& Q_{k}=Q\left(z^{k}, \mu_{k}\right)=\left(A_{k}^{\mathrm{T}} B_{k}^{-1} A_{k}\right)^{-1} A_{k}^{\mathrm{T}} B_{k}^{-1}, P_{k}=P\left(z^{k}, \mu_{k}\right)=B_{k}^{-1}\left(E_{n+2 m+1}-A_{k} Q_{k}\right) . \\
& \pi^{k}=\pi\left(z^{k}, \mu_{k}\right)=-Q\left(z^{k}, \mu_{k}\right) \nabla \theta_{c_{k}}\left(z^{k}, \mu_{k}\right), \\
& d_{0}^{k}=\left(d z_{0}^{k}, d \mu_{0}^{k}\right)=d_{0}\left(z^{k}, \mu_{k}\right)=-P_{k} \nabla \theta_{c_{k}}\left(z^{k}, \mu_{k}\right)+Q_{k}^{\mathrm{T}} V^{k} .
\end{aligned}
$$




$$
\begin{aligned}
& V^{k}=V\left(z^{k}, \mu_{k}\right)=\left(V_{i}^{k}, i \in L_{k}\right), V_{i}^{k}= \begin{cases}-r_{i}\left(z^{k}, \mu_{k}\right), & \pi_{i}^{k}>0, \\
\pi_{i}^{k}, & \pi_{i}^{k} \leq 0 .\end{cases} \\
& d_{1}^{k}=-Q_{k}^{\mathrm{T}}\left(\left\|d_{0}^{k}\right\|^{\tau} e+F\left(z^{k}+d z_{0}^{k}, \mu_{k}+d \mu_{0}^{k}\right)\right), d^{k}=\left(d z^{k}, d \mu_{k}\right)=d_{0}^{k}+d_{1}^{k} . \\
& \rho_{k}=-\nabla \theta_{c_{k}}\left(z^{k}, \mu_{k}\right)^{\mathrm{T}} d_{0}^{k}, d_{2}^{k}=\frac{-\rho_{k}}{1+2\left|e^{\mathrm{T}} \pi^{k}\right|} Q_{k}^{\mathrm{T}} e, q^{k}=\left(q z^{k}, q \mu_{k}\right)=\rho_{k}\left(d_{0}^{k}+d_{2}^{k}\right)
\end{aligned}
$$

where $e=(1, \cdots, 1)^{\mathrm{T}} \in R^{\left|L_{k}\right|}$.

According to the above analysis, the algorithm for the solution of the problem (1.1) can be stated as follows. Algorithm B:

Step 0. Given a starting point $\left(z^{1}, \mu_{1}\right) \in X_{1}$, and an initial symmetric positive definite matrix $B_{1} \in R^{(n+2 m+1) \times(n+2 m+1)}$. Choose parameters $\xi, \sigma, v, \varepsilon_{0} \in(0,1), \alpha \in\left(0, \frac{1}{2}\right), \tau \in(2,3), \delta_{0}>2, \delta_{1}>0, \delta_{2}>0, c_{k}>0, k=1$.

Step 1. By means of Algorithm A, compute $J_{k}=J\left(z^{k}, \mu_{k}\right), A_{k}=A\left(z^{k}, \mu_{k}\right)$ and $F\left(z^{k}, \mu_{k}\right)$.

Step 2. Compute $d_{0}^{k}$ according to (2.13). If $d_{0}^{k}=0$, stop; otherwise, compute $d^{k}$ according to (2.14). If

$$
\nabla \theta_{c_{k}}\left(z^{k}, \mu_{k}\right)^{\mathrm{T}} d_{0}^{k} \leq \min \left\{-\xi\left\|d_{0}^{k}\right\|^{\delta_{0}},-\xi\left\|d^{k}\right\|^{\delta_{0}}\right\}
$$

goto Step 3; otherwise, goto Step 4 .

Step 3. Let $\lambda=1$.

(1) If

$$
\begin{aligned}
& \theta_{c_{k}}\left(z^{k}+\lambda d z^{k}, \mu_{k}+\lambda d \mu_{k}\right) \leq \theta_{c_{k}}\left(z^{k}, \mu_{k}\right)+\alpha \lambda \nabla \theta_{c_{k}}\left(z^{k}, \mu_{k}\right)^{T} d_{0}^{k}, \\
& r_{i}\left(z^{k}+\lambda d z^{k}, \mu_{k}+d \mu_{k}\right) \leq 0, i \in T .
\end{aligned}
$$

Set $\lambda_{k}=\lambda$, goto Step 5 .

(2) Let $\lambda=\frac{1}{2} \lambda$. if $\lambda<\sigma$, goto Step 4; otherwise, repeat (1).

Step 4. Obtain feasible descent direction $q^{k}$ from (2.16), and compute $\beta_{k}$, the first number $\beta$ in the sequence $\left\{1, \frac{1}{2}, \frac{1}{4}, \cdots\right\}$ satisfying

$$
\begin{aligned}
& \theta_{c_{k}}\left(z^{k}+\beta q z^{k}, \mu_{k}+\beta q \mu_{k}\right) \leq \theta_{c_{k}}\left(z^{k}, \mu_{k}\right)+v \beta \nabla \theta_{c_{k}}\left(z^{k}, \mu_{k}\right)^{\mathrm{T}} q^{k}, \\
& r_{i}\left(z^{k}+\beta q z^{k}, \mu_{k}+\beta q \mu_{k}\right) \leq 0, i \in T .
\end{aligned}
$$

Let $d^{k}=q^{k}, \lambda_{k}=\beta_{k}$.

Step 5. Define $\tilde{\pi}^{k}=-\left(A_{k}^{\mathrm{T}} A_{k}\right)^{-1} A_{k}^{\mathrm{T}}\left(\begin{array}{c}\nabla f\left(s^{k}\right) \\ 0_{m \times 1} \\ 0\end{array}\right), \bar{c}\left(z^{k}, \mu_{k}\right)=\max \left\{-\tilde{\pi}_{i}^{k} \mid i \in T \backslash I_{1}\right\}+\delta_{1}$ and set

$$
c_{k+1}= \begin{cases}\max \left\{\bar{c}\left(z^{k}, \mu_{k}\right), c_{k}+\delta_{2}\right\}, & \bar{c}\left(z^{k}, \mu_{k}\right)>c^{k} \\ c_{k}, & \text { otherwise. }\end{cases}
$$

and $\left(z^{k+1}, \mu_{k+1}\right)=\left(z^{k}, \mu_{k}\right)+\lambda_{k} d^{k}$. Obtain $B_{k+1}$ by updating the positive definite matrix $B_{k}$ using some quasiNewton formulas, and set $k=k+1$. Go back to Step 1 .

In the remainder of this section, we give some results to show that Algorithm B is correctly stated. 
Lemma 2.2. (1) If $d_{0}^{k} \neq 0$, then we have

$$
\begin{aligned}
& \nabla \theta_{c_{k}}\left(z^{k}, \mu_{k}\right)^{\mathrm{T}} d_{0}^{k}<0, \quad \nabla \theta_{c_{k}}\left(z^{k}, \mu_{k}\right)^{\mathrm{T}} q^{k} \leq-\frac{1}{2} \rho_{k}^{2}<0, \\
& h_{i}\left(z^{k}, \mu_{k}\right)^{\mathrm{T}} d_{0}^{k} \leq 0, \quad h_{i}\left(z^{k}, \mu_{k}\right)^{\mathrm{T}} q^{k}<0, i \in I\left(z^{k}, \mu_{k}\right) .
\end{aligned}
$$

(2) If the sequence $\left\{z^{k}, \mu_{k}\right\}$ is bounded, then there exists a constant $c_{0}>0$ such that

$$
\nabla \theta_{c_{k}}\left(z^{k}, \mu_{k}\right)^{\mathrm{T}} q^{k} \leq c_{0}\left\|q^{k}\right\|^{2}
$$

Proof. (1) If $d_{0}^{k} \neq 0$, then

$$
\begin{aligned}
\nabla \theta_{c_{k}}\left(z^{k}, \mu_{k}\right)^{\mathrm{T}} d_{0}^{k} & =-\nabla \theta_{c_{k}}\left(z^{k}, \mu_{k}\right)^{\mathrm{T}} P_{k} \nabla \theta_{c_{k}}\left(z^{k}, \mu_{k}\right)-\left(\pi^{k}\right)^{\mathrm{T}} V^{k} \\
& =-\left(P_{k} \nabla \theta_{c_{k}}\left(z^{k}, \mu_{k}\right)\right)^{\mathrm{T}} B_{k}\left(P_{k} \nabla \theta_{c_{k}}\left(z^{k}, \mu_{k}\right)\right)-\sum_{\pi_{i}^{k} \leq 0}\left(\pi_{i}^{k}\right)^{2}+\sum_{\pi_{i}^{k}>0} \pi_{i}^{k} h_{i}^{k}<0, \\
\nabla \theta_{c_{k}}\left(z^{k}, \mu_{k}\right)^{\mathrm{T}} q^{k} & =\rho_{k} \nabla \theta_{c_{k}}\left(z^{k}, \mu_{k}\right)^{\mathrm{T}}\left(d_{0}^{k}+d_{2}^{k}\right)=\rho_{k}\left(-\rho_{k}+\frac{\rho_{k}}{1+2\left|\mathrm{e}^{\mathrm{T}} \pi^{k}\right|}\left(\pi^{k}\right)^{\mathrm{T}} e\right) \leq-\frac{1}{2} \rho_{k}^{2}<0 .
\end{aligned}
$$

In view of $A_{k}^{\mathrm{T}} d_{0}^{k}=V^{k}$, we get $\left(h_{i}^{k}\right)^{\mathrm{T}} d_{0}^{k} \leq 0, i \in I\left(z^{k}, \mu_{k}\right)$. Since

$$
A_{k}^{\mathrm{T}} q^{k}=\rho_{k} A_{k}^{\mathrm{T}}\left(d_{0}^{k}+d_{2}^{k}\right)=\rho_{k}\left(V^{k}-\frac{\rho_{k}}{1+2\left|e^{\mathrm{T}} \pi^{k}\right|} e\right) \leq \frac{-\rho_{k}^{2}}{1+2\left|e^{\mathrm{T}} \pi^{k}\right|} e,
$$

so we have

$$
\left(h_{i}^{k}\right)^{\mathrm{T}} q^{k} \leq \frac{-\rho_{k}^{2}}{1+2\left|e^{\mathrm{T}} \pi^{k}\right|}<0, i \in I\left(z^{k}, \mu_{k}\right) .
$$

(2) Note that the boundedness of sequence $\left\{z^{k}, \mu_{k}\right\}$ and $B_{k}$ positive definite, we know that $d_{0}^{k}, d_{2}^{k}$ are bounded. By (2.16), there exists constant $\hat{c}>0$ such that $\rho_{k} \geq \hat{c}\left\|q^{k}\right\|$. Thus, there exists constant $c_{0}>0$ such that

$$
\nabla \theta_{c_{k}}\left(z^{k}, \mu_{k}\right)^{\mathrm{T}} q^{k} \leq-\frac{1}{2} \rho_{k}^{2} \leq-c_{0}\left\|q^{k}\right\|^{2}
$$

So, the claim holds.

According to Lemma 2.2 and the continuity of functions $\theta_{c_{k}}\left(z^{k}, \mu_{k}\right)$ and $r_{i}\left(z^{k}, \mu_{k}\right), i \in T$, the following result is true.

Lemma 2.3. Algorithm B is well defined.

\section{Global Convergence}

In this section, we consider the global convergence of the algorithm B. Firstly, we show that $s^{k}$ is an exact stationary point of (1.1) if the Algorithm B terminates at the current iteration point $\left(z^{k}, \mu_{k}\right)$.

Lemma 3.1. (1) $\left(z^{k}, \mu_{k}\right)$ is a $K-T$ point of (1.5) if and only if $d_{0}^{k}=0$.

(2) If $\left(z^{k}, \mu_{k}\right)$ is a $K-T$ point of (1.5), then $\left(z^{k}, \mu_{k}\right)$ with $\mu_{k}=0$ is a $K-T$ point of (1.4).

Proof. (1) If $\left(z^{k}, \mu_{k}\right)$ is a $K-T$ point of (1.5), then from the definition of index set $J_{k}$, we know the $K-T$ multiplier corresponding to constraints about index $I_{1} \backslash J_{k}$ is 0 . Thus, there exists vector $\chi=\left(\chi_{i}, i \in L_{k}\right)$ such that

$$
\nabla \theta_{c_{k}}\left(z^{k}, \mu_{k}\right)+A_{k} \chi=0, \chi_{i} \geq 0, \chi_{i} r_{i}\left(z^{k}, \mu_{k}\right)=0, i \in L_{k} .
$$

Note that matrix $A_{k}$ is full of column rank, and $B_{k}$ positive definite. Thus we have $\left(A_{k}^{\mathrm{T}} B_{k}^{-1} A_{k}\right)^{-1}$ exists. 
Furthermore, it follows from (3.1) that

$$
\chi=-\left(A_{k}^{\mathrm{T}} B_{k}^{-1} A_{k}\right)^{-1} A_{k}^{\mathrm{T}} B_{k}^{-1} \nabla \theta_{c_{k}}\left(z^{k}, \mu_{k}\right)=-Q_{k} \nabla \theta_{c_{k}}\left(z^{k}, \mu_{k}\right)=\pi^{k} .
$$

By (2.14) and (3.1), we have

$$
V^{k}=0, B_{k}^{-1} \nabla \theta_{c_{k}}\left(z^{k}, \mu_{k}\right)-B_{k}^{-1} A_{k} Q_{k} \nabla \theta_{c_{k}}\left(z^{k}, \mu_{k}\right)=0, P_{k} \nabla \theta_{c_{k}}\left(z^{k}, \mu_{k}\right)=0,
$$

so $d_{0}^{k}=0$.

On the other hand, it is easy to verify that

$$
P_{k} A_{k}=0, P_{k} B_{k} P_{k}=P_{k}, Q_{k} A_{k}=E_{\left|L_{k}\right|} .
$$

It follows from $d_{0}^{k}=0$ that

$$
0=A_{k}^{\mathrm{T}} d_{0}^{k}=V^{k}, P_{k} \nabla \theta_{c_{k}}\left(z^{k}, \mu_{k}\right)=0 .
$$

From the positive definiteness of $B_{k}$ and (2.12), (2.13) and (2.14), we have

$$
\nabla \theta_{c_{k}}\left(z^{k}, \mu_{k}\right)+A_{k} \pi^{k}=0, \pi_{i}^{k} \geq 0, \pi_{i}^{k} r_{i}\left(z^{k}, \mu_{k}\right)=0, i \in L_{k},
$$

which implies that $\left(z^{k}, \mu_{k}\right)$ is a $K-T$ point of (1.5).

(2) In view of the definition of $\theta_{c}(z, \mu)$, we obtain from (3.2) that

$$
\begin{aligned}
& \left(\begin{array}{c}
\nabla f\left(s^{k}\right) \\
0_{m \times 1} \\
0
\end{array}\right)-c_{k} \sum_{i \in\left(T \backslash I_{1}\right)} h_{i}^{k}+\sum_{i \in L_{k}} \pi_{i}^{k} h_{i}^{k}=0, \\
& \pi_{i}^{k} \geq 0, \pi_{i}^{k} r_{i}\left(z^{k}, \mu_{k}\right)=0, i \in L_{k} .
\end{aligned}
$$

Since the vectors $\left\{h_{i}^{k}, i \in L_{k}\right\}$ are linearly independent, we have

$$
\pi_{L_{k}}^{k}=-\left(A_{k}^{\mathrm{T}} A_{k}\right)^{-1} A_{k}^{\mathrm{T}}\left(\left(\begin{array}{c}
\nabla f\left(s^{k}\right) \\
0_{m \times 1} \\
0
\end{array}\right)-c_{k} \sum_{i \in\left(T \backslash I_{1}\right)} h_{i}^{k}\right),
$$

i.e.

$$
\pi_{L_{k}}^{k}=\tilde{\pi}_{L_{k}}^{k}+c_{k} \sum_{i \in\left(T I_{1}\right)}\left(A_{k}^{\mathrm{T}} A_{k}\right)^{-1} A_{k}^{\mathrm{T}} h_{i}^{k}
$$

Thus, we deduce

$$
\pi_{i}^{k}=\tilde{\pi}_{i}^{k}+c_{k}, i \in\left(T \backslash I_{1}\right) .
$$

In view of the definition of penalty parameter $c_{k}$, from (3.4), we have

$$
\pi_{i}^{k}=\tilde{\pi}_{i}^{k}+c_{k} \geq \delta_{1}>0, i \in\left(T \backslash I_{1}\right) .
$$

Combining with (3.2) and (3.5), it holds that

$$
r_{i}\left(z^{k}, \mu_{k}\right)=0, i \in\left(T \backslash I_{1}\right), \mu_{k}=0 .
$$

Let $\bar{\pi}^{k}=\left(\bar{\pi}_{i}^{k}, i \in T\right)$, where $\bar{\pi}_{i}^{k}=\pi_{i}^{k}, i \in L_{k}, \bar{\pi}_{i}^{k}=0, i \in I_{1} \backslash J_{k}$. From (3.3) and (3.6), we can easily see that $\left(z^{k}, \mu_{k}, \bar{\pi}^{k}\right)$ is a $K-T$ point pair of (1.4).

Theorem 3.1. Suppose the nondegeneracy condition holds at $z^{k}$. If $\left(z^{k}, \mu_{k}\right)$ is a $K-T$ point of (1.4), then 
$s^{k}$ is a $K-T$ point of (1.1).

Proof. According to the $K-T$ system of (1.4) and the relationship of index $i$ and $j$ in (2.1), we see that

$$
\mu_{k}=0, w_{j}^{k}=G_{j}\left(s^{k}\right), \phi\left(t_{j}^{k}\right)=0, j \in I_{2} .
$$

Then, combining with Proposition 2.1 and Proposition 2.2, we can conclude that $s^{k}$ is a $K-T$ point of (1.1). In the sequel, it is assumed that the Algorithm B generates an infinite sequence $\left\{\left(z^{k}, \mu_{k}\right)\right\}$. The following further assumption about $\left\{\left(z^{k}, \mu_{k}\right)\right\}$ is required in subsequent discussions.

H 3.1. (1) The sequence $\left\{\left(z^{k}, \mu_{k}\right)\right\}$ is bounded.

(2) The accumulation point $\left(z^{*}, \mu_{*}\right)$ of infinite sequence $\left\{\left(z^{k}, \mu_{k}\right)\right\}$ satisfies (2.2).

From H 3.1 and the fact that there are only finitely many choices for sets $J_{k} \subseteq I_{1}$, we may assume that there exists a subsequence $K$, such that

$$
z^{k} \rightarrow z^{*}, B_{k} \rightarrow B_{*}, J_{k} \equiv J, k \in K,
$$

where $J$ is a constant set. Correspondingly, the following results hold:

$$
A_{k} \rightarrow A_{*}, Q_{k} \rightarrow Q_{*}, P_{k} \rightarrow P_{*}, d_{0}^{k} \rightarrow d_{0}^{*}, q^{k} \rightarrow q^{*}, k \in K, k \rightarrow \infty .
$$

Lemma 3.2. Suppose $\left(z^{k}, \mu_{k}\right) \rightarrow\left(z^{*}, \mu_{*}\right)$, then for $k \in K$ large enough, we have

(1) there exists a constant $\bar{\epsilon}>0$ such that $\epsilon_{k, l_{k}} \geq \bar{\epsilon}$.

(2) there exists a constant $c>0$ such that $c_{k} \equiv C$.

Proof. (1) suppose, by contradiction, that there exists an index set $K^{\prime} \subseteq K$ such that $\epsilon_{k, l_{k}} \rightarrow 0\left(k \in K^{\prime}, k \rightarrow \infty\right)$. Let $J_{k^{\prime}}=J_{k, l-1}$. For $k \in K^{\prime}$ large enough, from Algorithm A, we have

$$
\operatorname{det}\left(A_{J_{k}^{\prime} \cup\left(T \backslash I_{1}\right)}^{\mathrm{T}} A_{J_{k}^{\prime} \cup\left(T \backslash I_{1}\right)}\right)<2 \epsilon_{k, l_{k}},-\epsilon_{k, l}\left|\varrho_{i}\left(z^{k}, \mu_{k}\right)\right| \leq r_{i}\left(z^{k}, \mu_{k}\right) \leq 0, \quad i \in J_{k}^{\prime} .
$$

Since there are only finite possible subsets of $I_{1}$, there must be an infinite subset $K^{\prime \prime} \in K^{\prime}$ such that for any $k \in K^{\prime \prime}, J_{k}^{\prime}=J^{\prime}$. Thus, it follows from (3.9) that

$$
\begin{aligned}
& \operatorname{det}\left(A_{J^{\prime} \cup\left(T \backslash \Lambda_{1}\right)}^{\mathrm{T}} A_{J^{\prime} \cup\left(T \backslash I_{1}\right)}\right)<2 \epsilon_{k, l_{k}} \rightarrow 0, k \in K^{\prime \prime}, k \rightarrow \infty, \\
& r_{i}\left(z^{*}, \mu_{*}\right)=0, i \in J^{\prime},
\end{aligned}
$$

which contradicts the condition $\mathrm{H} 2.3$.

(2) Suppose by contradiction, there exists a subsequence $\left\{k_{i}\right\}$ such that $c_{k_{i}}>c_{k_{i}-1}(i=1,2, \cdots)$, then from the definition of $c_{k}$, we have

$$
\begin{aligned}
& \bar{c}\left(z^{k}, \mu_{k}\right)>c_{k_{i}-1}(i=1,2, \cdots), \\
& c_{k} \rightarrow \infty, k \rightarrow \infty .
\end{aligned}
$$

From the finite selectivity of $J_{k}$, we can suppose without loss of generality that $J_{k_{i}}=J(i=1,2, \cdots)$. By (1), we can see that $\bar{c}\left(z_{k_{i}}, \mu_{k_{i}}\right)$ is bounded, i.e., $\bar{c}\left(z_{k_{i}}, \mu_{k_{i}}\right)<c^{*}(i=1,2, \cdots)$ for some $c^{*}$. Let $M$ be such an integer that $c_{k_{i}-1} \geq c^{*}(i \geq M)$, then we have

$$
c_{k_{i}-1} \geq c^{*} \geq \bar{c}\left(z_{k_{i}}, \mu_{k_{i}}\right)(i \geq M),
$$

a contradiction, and the result is proved.

Lemma 3.3. Suppose that $\left(z^{k}, \mu_{k}\right) \rightarrow\left(z^{*}, \mu_{*}\right)(k \in K)$, and $\left(z^{k+1}, \mu_{k+1}\right)=\left(z^{k}, \mu_{k}\right)+\lambda_{k} d^{k}(k \in K)$ which is generated by Step 4 and Step 5. If $\left(z^{*}, \mu_{*}\right)$ is not a $K-T$ point of (1.5), then we have

(1) $\nabla \theta_{c}\left(z^{*}, \mu_{*}\right)^{\mathrm{T}} q^{*}<0, h_{i}\left(z^{*}, \mu_{*}\right)^{\mathrm{T}} q^{*}, i \in I\left(z^{*}, \mu_{*}\right)$,

(2) $\beta_{k} \geq \beta_{*}=\inf \left\{\beta_{k}, k \in K\right\}>0, k \in K$.

Proof. (1) Suppose $B_{k} \rightarrow B_{*}, d_{0}^{k} \rightarrow d_{0}^{*}, q^{k} \rightarrow q^{*}, k \in K$. Since $\left(z^{*}, \mu_{*}\right)$ is not a $K-T$ of (1.5), so we have $d_{0}^{*} \neq 0$ and 


$$
\begin{aligned}
& \nabla \theta_{c}\left(z^{*}, \mu_{*}\right)^{\mathrm{T}} d_{0}^{*}<0, \nabla \theta_{c}\left(z^{*}, \mu_{*}\right)^{\mathrm{T}} q^{*}<0, \\
& h_{i}\left(z^{*}, \mu_{*}\right)^{\mathrm{T}} q^{*}<0, i \in I\left(z^{*}, \mu_{*}\right) .
\end{aligned}
$$

Therefore, for $k \in K$ large enough, we obtain

$$
\begin{aligned}
& -\rho_{k} \leq \frac{1}{2} \nabla \theta_{c}\left(z^{*}, \mu_{*}\right)^{\mathrm{T}} d_{0}^{*}<0, \\
& \nabla \theta_{c}\left(z^{k}, \mu_{k}\right)^{\mathrm{T}} q^{k} \leq \nabla \frac{1}{2} \theta_{c}\left(z^{*}, \mu_{*}\right)^{\mathrm{T}} d_{0}^{*}<0, \\
& h_{i}\left(z^{k}, \mu_{k}\right)^{T} q^{k}<0, i \in I\left(z^{*}, \mu_{*}\right) .
\end{aligned}
$$

(2) For (2.20), denote

$$
\begin{aligned}
a & \triangleq \theta_{c}\left(z^{k}+\beta q z^{k}, \mu_{k}+\beta q \mu_{k}\right)-\theta_{c}\left(z^{k}, \mu_{k}\right)-v \beta \nabla \theta_{c}\left(z^{k}, \mu_{k}\right)^{\mathrm{T}} q^{k} \\
& =(1-v) \beta \nabla \theta_{c}\left(z^{k}, \mu_{k}\right)^{\mathrm{T}} q^{k}+o(\beta) .
\end{aligned}
$$

From (3.12), for $k \in K$ large enough and $\beta>0$ small enough, it holds that $s \leq 0$.

For (2.21), when $i \in T \backslash I\left(z^{*}, \mu_{*}\right)$, the fact $\left(z^{k}, \mu_{k}\right) \rightarrow\left(z^{*}, \mu_{*}\right), r_{i}\left(z^{*}, \mu_{*}\right)<0, k \in K$ and the continuity of $r_{i}$ imply that (4.5) holds. When $i \in I\left(z^{*}, \mu_{*}\right)$, it holds that $r_{i}\left(z^{*}, \mu_{*}\right)=0$. From (3.12), for $k \in K$ large enough and $\beta>0$ small enough, we have

$$
\begin{aligned}
r_{i}\left(z^{k}+\beta q z^{k}, \mu_{k}+\beta q \mu_{k}\right) & =r_{i}\left(z^{k}, \mu_{k}\right)+\beta h_{i}\left(z^{k}, \mu_{k}\right)^{\mathrm{T}} q^{k}+o(\beta) \\
& \leq \beta h_{i}\left(z^{k}, \mu_{k}\right)^{\mathrm{T}} q^{k}+o(\beta) \leq 0 .
\end{aligned}
$$

According to the analysis above, the result is true.

Lemma 3.4. Algorithm $B$ generates infinite sequence $\left\{\left(z^{k}, \mu_{k}\right)\right\}$, whose any accumulation points $\left(z^{*}, \mu_{*}\right)$ are $K-T$ points of (1.1).

Proof. Suppose that $\left\{\left(z^{k}, \mu_{k}\right)\right\} \rightarrow\left(z^{*}, \mu_{*}\right), k \in K$. From (2.17), (2.18), (2.20) and Lemma 2.2, we know that $\left\{\theta_{c}\left(z^{k}, \mu_{k}\right)\right\}$ is a descent sequence. While, for $k \in K, k \rightarrow \infty$, it is obvious that $\theta_{c}\left(z^{k}, \mu_{k}\right) \rightarrow \theta_{c}\left(z^{*}, \mu_{*}\right)$. So

$$
\theta_{c}\left(z^{k}, \mu_{k}\right) \rightarrow \theta_{c}\left(z^{*}, \mu_{*}\right), k \rightarrow \infty .
$$

Now we consider the following two cases:

(1) Suppose there exists an infinite subset $K_{1} \subseteq K$ such that

$$
\left(z^{k+1}, \mu_{k+1}\right)=\left(z^{k}, \mu_{k}\right)+\lambda_{k} d^{k},
$$

which is obtained by Step 3 and Step 5. In view of $\lambda_{k} \geq \sigma, k \in K_{1}$ in Step 3, it follows from (2.17) and (2.18) that

$$
\lim _{k \in K_{1}}\left(\theta_{c}\left(z^{k+1}, \mu_{k+1}\right)-\theta_{c}\left(z^{k}, \mu_{k}\right)\right) \leq \lim _{k \in K_{1}} \alpha \lambda \nabla \theta_{c}\left(z^{k}, \mu_{k}\right)^{\mathrm{T}} d_{0}^{k} \leq \lim _{k \in K_{1}}\left(-\alpha \varepsilon \xi\left\|d_{0}^{k}\right\|^{\delta_{0}}\right) \leq 0 .
$$

Obvious, $d_{0}^{k} \rightarrow 0, k \rightarrow K_{1}$. Again, $d_{0}^{k} \rightarrow d_{0}^{*}, k \in K$, so we have $d_{0}^{*}=0$. Imitating the proof of Lemma 3.1, it is easy to see that $\left(z^{*}, \mu_{*}\right)$ is a $K-T$ point of (1.5).

(2) Assume the iteration $\left(z^{k+1}, \mu_{k+1}\right)=\left(z^{k}, \mu_{k}\right)+\lambda_{k} d^{k}, \forall k \in K$ is generated by Step 4 and Step 5 . Suppose by contradiction that $\left(z^{*}, \mu_{*}\right)$ is not a $K-T$ point of (1.5). Then, from (3.12) and Lemma 3.3, we have

$$
0=\lim _{k \in K}\left(\theta_{c}\left(z^{k+1}, \mu_{k+1}\right)-\theta\left(z^{k}, \mu_{k}\right)\right) \leq \lim _{k \in K} v \beta_{k} \nabla \theta_{c}\left(z^{k}, \mu_{k}\right)^{\mathrm{T}} q^{k} \leq \frac{1}{2} \nu \beta_{*} \nabla \theta_{c}\left(z^{*}, \mu_{*}\right)^{\mathrm{T}} q^{*}<0,
$$

which is a contradiction. Thus, the claim holds. 
Theorem 3.2. The $K-T$ point $\left(z^{*}, \mu_{*}\right)$ of (1.5) must be the one of (1.4), where $\mu_{*}=0$. Proof. If $\left(z^{*}, \mu_{*}\right)$ is a $K-T$ point of (1.5), then there exists multiplier $\pi^{*}$ such that

$$
\begin{aligned}
& \left(\begin{array}{c}
\nabla f\left(s^{*}\right) \\
0_{m \times 1} \\
0
\end{array}\right)-c_{*} \sum_{i \in\left(T \backslash I_{1}\right)} h_{i}^{*}+\sum_{i \in\left(J \cup\left(T I_{1}\right)\right)} \pi_{i}^{*} h_{i}^{*}=0, \\
& \pi_{i}^{*} \geq 0, \pi_{i}^{*} r_{i}\left(z^{*}, \mu_{*}\right)=0, i \in J \cup\left(T \backslash I_{1}\right) .
\end{aligned}
$$

Set

$$
A_{*}=\left(h_{i}^{*} \mid i \in J \cup\left(T \backslash I_{1}\right)\right), \tilde{\pi}^{*}=-\left(A_{*}^{\mathrm{T}} A_{*}\right)^{-1} A_{*}^{\mathrm{T}}\left(\begin{array}{c}
\nabla f\left(s^{*}\right) \\
0_{m \times 1} \\
0
\end{array}\right) .
$$

Obvious, $\tilde{\pi}_{J \cup\left(T I_{1}\right)}^{k} \rightarrow \tilde{\pi}_{J \cup\left(T \backslash I_{1}\right)}^{*}$. While, from (3.14) we get

$$
\pi_{J \cup\left(T \backslash I_{1}\right)}^{*}=-\left(A_{*}^{\mathrm{T}} A\right)^{-1} A_{*}^{\mathrm{T}}\left(\left(\begin{array}{c}
\nabla f\left(s^{*}\right) \\
0_{m \times 1} \\
0
\end{array}\right)-c_{*} \sum_{i \in\left(T \cup_{1}\right)} h_{i}^{*}\right)
$$

i.e.

$$
\pi_{J \cup\left(T \backslash I_{1}\right)}^{*}=\tilde{\pi}_{J \cup\left(T \backslash_{1}\right)}^{*}+c_{*} \sum_{i \in\left(T \backslash \backslash_{1}\right)}\left(A_{*}^{\mathrm{T}} A_{*}\right)^{-1} A_{*}^{\mathrm{T}} h_{i}^{*} .
$$

Thereby,

$$
\pi_{i}^{*}=\tilde{\pi}_{i}^{*}+C_{*}, i \in\left(T \backslash I_{1}\right) .
$$

According to the definition of $c_{*}$, it is clear that

$$
\pi_{i}^{*}=\tilde{\pi}_{i}^{*}+c_{*} \geq \delta_{1}>0, i \in\left(T \backslash I_{1}\right) .
$$

In addition, combining with (3.2) (3.16), we obtain

$$
r_{i}\left(z^{*}, \mu_{*}\right)=0, i \in\left(T \backslash I_{1}\right), \mu_{*}=0 .
$$

Let $\bar{\pi}^{*}=\left(\bar{\pi}_{i}^{*}, i \in T\right)$, where $\bar{\pi}_{i}^{*}=\pi_{i}^{*}, i \in J \cup\left(T \backslash I_{1}\right), \bar{\pi}_{i}^{*}=0, i \in I_{1} \backslash J$. It follows from (3.14) and (3.17) that $\left(z^{*}, 0, \bar{\pi}^{*}\right)$ is a $K-T$ point pair of (1.4).

Theorem 3.3. Suppose (2.2) holds at $z^{*}$. If $\left(z^{*}, 0\right)$ is a $K-T$ point of (1.4), then $s^{*}$ is a $K-T$ point of (1.1).

Proof. According to Theorem 3.2 and (2.1), Proposition 2.1 and Proposition 2.2 imply $s^{*}$ is a $K-T$ point of (1.1).

\section{Superlinear Convergence}

Now we discuss the convergence rate of the Algorithm B, and prove that the sequence $\left(z^{k}, \mu_{k}\right)$ generated by the Algorithm B is one-step superlinearly convergent. For this purpose, we add some stronger regularity assumptions.

H 4.1. The bounded sequence $\left\{\left(z^{k}, \mu_{k}\right)\right\}$ possesses an accumulation point $\left(z^{*}, \mu_{*}\right)$, at which second-order sufficiency condition and strict complementary slackness hold, where $\zeta^{*}=\left(\zeta_{i}^{*}, i \in T\right)$ is the corresponding multiplier of $\left(z^{*}, \mu_{*}\right)$. 
Lemma 4.1. Under $H$ 2.1-H 4.2, we have that

$$
\lim _{k \rightarrow \infty}\left\|z^{k+1}-z^{k}\right\|=0, \lim _{k \rightarrow \infty}\left(\mu_{k+1}-\mu_{k}\right)=0 .
$$

Proof. For $\left(z^{k+1}, \mu_{k+1}\right)=\left(z^{k}, \mu_{k}\right)+\lambda_{k} d^{k}$ generated by Step 3 and Step 5, from (2.17) and (2.18), it holds that

$$
\theta_{c}\left(z^{k}+\lambda_{k} d z^{k}, \mu_{k}+\lambda_{k} d \mu_{k}\right) \leq \theta_{c}\left(z^{k}, \mu_{k}\right)+\alpha \lambda_{k} \nabla \theta_{c}\left(z^{k}, \mu_{k}\right)^{\mathrm{T}} d_{0}^{k} \leq \theta_{c}\left(z^{k}, \mu_{k}\right)+\alpha \xi \lambda_{k}\left\|d^{k}\right\|^{\delta_{0}} .
$$

While, for $\left(z^{k+1}, \mu_{k+1}\right)=\left(z^{k}, \mu_{k}\right)+\lambda_{k} d^{k}$ generated by Step 4 and Step 5, from (2.17), (2.20) and Lemma 2.2, we have

$$
\theta_{c}\left(z^{k}+\lambda_{k} d z^{k}, \mu_{k}+\lambda_{k} d \mu_{k}\right) \leq \theta_{c}\left(z^{k}, \mu_{k}\right)+v \lambda_{k} \nabla \theta_{c}\left(z^{k}, \mu_{k}\right)^{\mathrm{T}} d_{0}^{k} \leq \theta_{c}\left(z^{k}, \mu_{k}\right)-c_{0} v \lambda_{k}\left\|d^{k}\right\|^{2}
$$

So

$$
\theta_{c}\left(z^{k+1}, \mu_{k+1}\right) \leq \theta_{c}\left(z^{k}, \mu_{k}\right)-\lambda_{k}\left\|d^{k}\right\|^{2} \min \left\{c_{0} v, \alpha \xi\left\|d^{k}\right\|^{\delta_{0}-2}\right\}, \forall k .
$$

Passing to the limit $k \rightarrow \infty$ and from (3.13), we obtain

$$
\lim _{k \rightarrow \infty} \lambda_{k}\left\|d^{k}\right\|^{2} \min \left\{c_{0} v, \alpha \xi\left\|d^{k}\right\|^{\delta_{0}-2}\right\}=0
$$

Thereby

$$
\lim _{k \rightarrow \infty}\left\|z^{k+1}-z^{k}\right\|=0, \lim _{k \rightarrow \infty}\left(\mu_{k+1}-\mu_{k}\right)=0 .
$$

Theorem 4.1. The entire sequence $\left(z^{k}, \mu_{k}\right)$ converges to $\left(z^{*}, 0\right)$, i.e., $\left(z^{k}, \mu_{k}\right) \rightarrow\left(z^{*}, 0\right), k \rightarrow \infty$. In order to obtain the superlinear convergence rate, we make the following assumption.

H 4.2. $B_{k} \rightarrow B_{*}, k \rightarrow \infty, B_{*}$ positive definite.

Lemma 4.2. If $H$ 2.1- $H 4.2$ hold, then we get that

(1) for $k$ large enough, $L_{k}=I\left(z^{*}, \mu_{*}\right)=I_{*}$.

(2) $\lim _{k \rightarrow \infty} d_{0}^{k}=0, \lim _{k \rightarrow \infty} \pi^{k}=\left(\zeta_{i}^{*}, i \in\left(I_{*} \cup\left(T \backslash I_{1}\right)\right)\right.$.

Proof. (1) On one hand, by Lemma 3.2, for $k$ large enough, there exists a constant $\bar{\epsilon}>0$ such that $\bar{\epsilon} \leq \epsilon_{k, l_{k}}<1$ in Algorithm A. It follows from H 4.1 and the fact $\varrho\left(z^{k}, \mu_{k}\right) \rightarrow \zeta^{*}$ that, for $k$ large enough, $I_{*} \subseteq L_{k}$.

On the other hand, we assert that $L_{k} \subseteq I_{*}$. Otherwise, there exists some index $t$ and infinite subset $K$ such that

$$
t \in L_{k} \backslash I_{*}, r_{t}\left(z^{*}, 0\right)<0, r_{t}\left(z^{k}, \mu_{k}\right) \geq-\epsilon_{k, l_{k}}\left|\mu_{t}\left(z^{k}, \mu_{k}\right)\right| \geq-\left|\mu_{t}\left(z^{k}, \mu_{k}\right)\right|, \forall k \in K .
$$

Let $k \in K, k \rightarrow \infty$, then

$$
0>r_{t}\left(z^{*}, 0\right) \geq-\left|\mu_{t}\left(z^{*}, 0\right)\right|=-\zeta_{t}^{*}, \zeta_{t}^{*}>0 .
$$

It is a contradiction with the complementary slackness condition, which shows that $L_{k} \subseteq I_{*}$, i.e., $L_{k_{*}}=I_{*}$.

(2) According to $\left(z^{k}, \mu_{k}\right) \rightarrow\left(z^{*}, 0\right)$ and $B_{k} \rightarrow B_{*}$, the fact $L_{k}=I_{*}$ implies $d_{0}^{k} \rightarrow d_{0}^{*}, \pi^{k} \rightarrow \pi^{*}, k \rightarrow \infty$. Again, since $\left(z^{*}, 0\right)$ is a $K-T$ point of (1.5), imitating the proof of Lemma 3.1, we get that

$$
d_{0}^{*}=0, \quad \nabla \theta_{c}\left(z^{*}, \mu_{*}\right)+A_{*} \pi^{*}=0, \quad \pi_{i}^{*} r_{i}=0, \quad \pi_{i}^{*} \geq 0, \quad i \in I_{*} .
$$

So the uniqueness of $K-T$ multiplier shows $\lim _{k \rightarrow \infty} \pi^{k}=\zeta^{*}$.

Lemma 4.3. Under $H$ 2.1-H 4.2, for $k$ large enough, $d_{0}^{k}$ with the corresponding multiplier $\zeta^{k}=\pi^{k}+\left(A_{k}^{\mathrm{T}} B_{k}^{-1} A_{k}\right)^{-1} F\left(z^{k}, \mu_{k}\right)$ is a $K-T$ point of the following quadratic program 


$$
\begin{array}{ll}
\min & \nabla \theta_{c}\left(z^{k}, \mu_{k}\right)^{\mathrm{T}} d+\frac{1}{2} d^{\mathrm{T}} B_{k} d \\
\text { s.t. } & r_{i}\left(z^{k}, \mu_{k}\right)+\left(h_{i}^{k}\right)^{\mathrm{T}} d=0, \quad i \in I_{*} .
\end{array}
$$

Proof. Suppose that $(d, \zeta)$ is a $K-T$ point pair of (4.1). From (2.12), (2.14) and (4.1), it holds that

$$
d=-P_{k} \nabla \theta_{c}\left(z^{k}, \mu_{k}\right)-Q_{k}^{\mathrm{T}} F\left(z^{k}, \mu_{k}\right), \quad \zeta=\pi^{k}+\left(A_{k}^{\mathrm{T}} B_{k}^{-1} A_{k}\right)^{-1} F\left(z^{k}, \mu_{k}\right)=\zeta^{k} .
$$

In addition, for $k$ large enough, $\pi_{i}^{k}>0, i \in I_{*}$ holds from fact $\lim _{k \rightarrow \infty} \pi^{k}=\zeta^{*}$ and strict complementarity condition. While, from the definition of $V^{k}$, it holds that $d_{0}^{k}=-P_{k} \nabla \theta_{c}\left(z^{k}, \mu_{k}\right)-Q_{k}^{\mathrm{T}} F\left(z^{k}, \mu_{k}\right)=d$. So the claim holds.

Lemma 4.4. (1) For $k$ large enough, there exist constants $b, \eta>0$ such that

$$
\sum_{i \in I+I\left(T \backslash I_{1}\right)} \zeta_{i}^{k} r_{i}^{k} \leq \eta\left\|F\left(z^{k}, \mu_{k}\right)\right\|, \quad \nabla \theta_{c}\left(z^{k}, \mu_{k}\right)^{T} d_{0}^{k} \leq-b\left\|d_{0}^{k}\right\|^{2} .
$$

(2) $d^{k}=d_{0}^{k}+d_{1}^{k}$ obtained by (2.15) satisfies

$$
\left\|d^{k}\right\| \sim\left\|d_{0}^{k}\right\|, \quad\left\|d_{1}^{k}\right\| \sim O\left(\left\|d_{0}^{k}\right\|^{2}\right) .
$$

Proof. (1) Since $\left(z^{k}, \mu_{k}\right) \rightarrow\left(z^{*}, \mu_{*}\right)$, and for $k$ large enough, $L_{k}=I_{*}$, it is easy to see

$$
F\left(z^{k}, \mu_{k}\right) \rightarrow\left(r_{i}\left(z^{*}, \mu_{*}\right), i \in I_{*}\right)=0, \quad \zeta^{k} \rightarrow \pi^{*}>0, \quad k \rightarrow \infty .
$$

Obviously, for $k$ large enough, $\zeta_{i}^{k}>0, i \in I_{*}$. Thereby, there exists a constant $\eta>0$ such that

$$
\sum_{i \in I_{*}} \zeta_{i}^{k} r_{i}^{k}=-\sum_{i \in I_{*}} \zeta_{i}^{k}\left|r_{i}^{k}\right| \leq-\eta\left\|F\left(z^{k}, \mu_{k}\right)\right\| .
$$

In addition, from Lemma 4.3, we see

$$
\nabla \theta_{c}\left(z^{k}, \mu_{k}\right)+B_{k} d_{0}^{k}+A_{k} \zeta^{k}=0, \quad F\left(z^{k}, \mu_{k}\right)+A_{k}^{\mathrm{T}} d_{0}^{k}=0 .
$$

So

$$
\begin{aligned}
\nabla \theta_{c}\left(z^{k}, \mu_{k}\right)^{\mathrm{T}} d_{0}^{k} & =-\left(d_{0}^{k}\right)^{\mathrm{T}} B_{k} d_{0}^{k}-\left(A_{k}^{\mathrm{T}} d_{0}^{k}\right)^{\mathrm{T}} \zeta^{k}=-\left(d_{0}^{k}\right)^{\mathrm{T}} B_{k} d_{0}^{k}+\sum_{i \in I_{*}} \zeta_{i}^{k} r_{i}^{k} \\
& \leq-b\left\|d_{0}^{k}\right\|^{2}-\eta\left\|F\left(z^{k}, \mu_{k}\right)\right\| \leq-b\left\|d_{0}^{k}\right\|^{2} .
\end{aligned}
$$

(2) Since

$$
r_{i}\left(z^{k}+d z_{0}^{k}, \mu_{k}+d \mu_{0}^{k}\right)=r_{i}\left(z^{k}\right)+\left(h_{i}^{k}\right)^{\mathrm{T}} d_{0}^{k}+O\left(\left\|d_{0}^{k}\right\|^{2}\right), \forall i \in I_{*},
$$

we know

$$
\left\|F\left(z^{k}+d z_{0}^{k}, \mu_{k}+d \mu_{0}^{k}\right)\right\|=O\left(\left\|d_{0}^{k}\right\|^{2}\right) .
$$

From $\tau \in(2,3), Q_{k} \rightarrow Q_{*}$ and the boundedness of $Q_{*}$, it follows that

$$
\left\|d^{k}\right\| \sim\left\|d_{0}^{k}\right\|,\left\|d_{1}^{k}\right\| \sim O\left(\left\|d_{0}^{k}\right\|^{2}\right) \text {. }
$$

So, the result is true.

In order to obtain the superlinear convergence rate, we make another assumption.

$\mathbf{H}$ 4.3. The sequence of symmetric matrices $\left\{B_{k}\right\}$ satisfies 


$$
\left\|\bar{P}_{k}\left(B_{k}-\nabla^{2} L\left(z^{k}, \mu_{k}, \zeta^{k}\right)\right) d_{0}^{k}\right\|=o\left(\left\|d_{0}^{k}\right\|\right) \Leftrightarrow\left\|\bar{P}_{k}\left(B_{k}-\nabla^{2} L\left(z^{*}, 0, \zeta^{*}\right)\right) d_{0}^{k}\right\|=o\left(\left\|d_{0}^{k}\right\|\right),
$$

where

$$
\begin{aligned}
& \bar{P}_{k}=E_{n+2 m+1}-A_{k}\left(A_{k}^{\mathrm{T}} A_{k}\right)^{-1} A_{k}^{\mathrm{T}}, \\
& \nabla^{2} L\left(z^{k}, \mu_{k}, \zeta^{k}\right)=\nabla^{2} \theta_{c_{k}}\left(z^{k}, \mu_{k}\right)+\sum_{i \in I_{*}} \zeta_{i}^{k} H_{i}^{k}, \\
& \nabla^{2} L\left(z^{*}, 0, \zeta^{*}\right)=\nabla^{2} \theta_{c}\left(z^{*}, 0\right)+\sum_{i \in T} \zeta_{i}^{*} H_{i}^{*} .
\end{aligned}
$$

Lemma 4.5. For k large enough, Algorithm B is not implemented on Step 4, and

$$
\lambda \equiv 1, \quad\left(z^{k+1}, \mu_{k+1}\right)=\left(z^{k}, \mu_{k}\right)+\lambda d^{k}
$$

holds in Step 3.

Proof. According to $d_{0}^{k} \rightarrow 0$ and Lemma 4.4, we have

$$
\left\|d_{0}^{k}\right\| \sim d^{k}\left\|, \quad \nabla \theta_{c}\left(z^{k}, \mu_{k}\right)^{T} d_{0}^{k} \leq-b\right\| d_{0}^{k} \|^{2},
$$

which shows (2.17) hold. Now we prove that, the arc search (2.19) and (2.18) eventually accept unit step, i.e., $\lambda_{k}=1$, for $k$ large enough.

Firstly, for (2.19), when $i \in T \backslash I_{*}$, the fact that $d_{0}^{k} \rightarrow 0, z^{k} \rightarrow z^{*}, \mu_{k} \rightarrow 0, r_{i}\left(z^{*}, 0\right)<0$ and the continuity of $r_{i}$ imply

$$
r_{i}\left(z^{k}+d z^{k}, \mu_{k}+d \mu_{k}\right) \leq 0
$$

when $i \in I_{*}$, using Taylor expansion, we get

$$
\begin{aligned}
& r_{i}\left(z^{k}+d z_{0}^{k}+d z_{1}^{k}, \mu_{k}+d \mu_{0}^{k}+d \mu_{1}^{k}\right) \\
& =r_{i}\left(z^{k}+d z_{0}^{k}, \mu_{k}+d \mu_{0}^{k}\right)+h_{i}\left(z^{k}+d z_{0}^{k}, \mu_{k}+d \mu_{0}^{k}\right)^{\mathrm{T}} d_{1}^{k}+O\left(\left\|d_{1}^{k}\right\|^{2}\right) \\
& =r_{i}\left(z^{k}+d z_{0}^{k}, \mu_{k}+d \mu_{0}^{k}\right)+h_{i}\left(z^{k}, \mu_{k}\right)^{\mathrm{T}} d_{1}^{k}+O\left(\left\|d_{0}^{k}\right\|^{3}\right) .
\end{aligned}
$$

Again, from

$$
A_{k}^{\mathrm{T}} d_{1}^{k}=-\left\|d_{0}^{k}\right\|^{\tau} e-F\left(z^{k}+d z_{0}^{k}, \mu_{k}+d \mu_{0}^{k}\right)
$$

we see

$$
h_{i}\left(z^{k}, \mu_{k}\right)^{\mathrm{T}} d_{1}^{k}=-\left\|d_{0}^{k}\right\|^{\tau}-r_{i}\left(z^{k}+d z_{0}^{k}, \mu_{k}+d \mu_{0}^{k}\right), i \in I_{*}
$$

Thus, (4.7) yields

$$
r_{i}\left(z^{k}+d z^{k}, \mu_{k}+d \mu^{k}\right)=-\left\|d_{0}^{k}\right\|^{\tau}+O\left(\left\|d_{0}^{k}\right\|^{3}\right) .
$$

In view of $\tau \in(2,3)$, (2.19) obviously holds when $\lambda_{k}=1$.

Secondly, we prove that, for $k$ large enough, (2.18) holds for $\lambda_{k}=1$. Denote

$$
\begin{aligned}
\varphi & =\theta_{c_{k}}\left(z^{k}+\lambda d z^{k}, \mu_{k}+\lambda d \mu_{k}\right)-\theta_{c_{k}}\left(z^{k}, \mu_{k}\right)-\alpha \nabla \theta_{c_{k}}\left(z^{k}, \mu_{k}\right)^{\mathrm{T}} d_{0}^{k}, \\
& =\nabla \theta_{c_{k}}\left(z^{k}, \mu_{k}\right)^{\mathrm{T}} d^{k}+\frac{1}{2}\left(d_{0}^{k}\right)^{\mathrm{T}} \nabla^{2} \theta_{c_{k}}\left(z^{k}, \mu_{k}\right)^{\mathrm{T}} d_{0}^{k}-\alpha \nabla \theta_{c_{k}}\left(z^{k}, \mu_{k}\right)^{\mathrm{T}} d_{0}^{k}+\left(\left\|d_{0}^{k}\right\|^{2}\right) .
\end{aligned}
$$

From (4.5), we have 


$$
\nabla \theta_{c_{k}}\left(z^{k}, \mu_{k}\right)^{\mathrm{T}} d^{k}=-\left(d_{0}^{k}\right)^{\mathrm{T}} \nabla^{2} \theta_{c_{k}}\left(z^{k}, \mu_{k}\right)^{\mathrm{T}} d_{0}^{k}-\sum_{i \in I_{*}} \zeta_{i}^{k}\left(h_{i}^{k}\right)^{\mathrm{T}} d^{k}+o\left(\left\|d_{0}^{k}\right\|^{3}\right) .
$$

Also, by (4.8), it holds that

$$
r_{i}^{k}+\left(h_{i}^{k}\right)^{\mathrm{T}} d^{k}+\frac{1}{2}\left(d_{0}^{k}\right)^{\mathrm{T}} H_{i}^{k}\left(d_{0}^{k}\right)+o\left(\left\|d_{0}^{k}\right\|^{2}\right)=-\left\|d_{0}^{k}\right\|^{\tau}+O\left(\left\|d_{0}^{k}\right\|^{3}\right), i \in I_{*} .
$$

So

$$
-\sum_{i \in I_{*}} \zeta_{i}^{k}\left(h_{i}^{k}\right)^{\mathrm{T}} d^{k}=-\sum_{i \in I_{*}} \zeta_{i}^{k} r_{i}^{k}+\frac{1}{2}\left(d_{0}^{k}\right)^{\mathrm{T}}\left(\sum_{i \in I_{*}} \zeta_{i}^{k} H_{i}^{k}\right) d_{0}^{k}+o\left(\left\|d_{0}^{k}\right\|^{2}\right)
$$

Thus, (4.6) yields

$$
\begin{aligned}
\varphi & =(\alpha-1)\left(d_{0}^{k}\right)^{\mathrm{T}} B_{k} d_{0}^{k}+\frac{1}{2}\left(d_{0}^{k}\right)^{\mathrm{T}} \nabla^{2} L\left(z^{k}, \mu_{k}, \zeta^{k}\right) d_{0}^{k}+\sum_{i \in I_{*}}(1-\alpha) \zeta_{i}^{k} r_{i}^{k}+o\left(\left\|d_{0}^{k}\right\|^{2}\right) \\
& \leq(\alpha-1)\left(d_{0}^{k}\right)^{\mathrm{T}} B_{k} d_{0}^{k}+\frac{1}{2}\left(d_{0}^{k}\right)^{\mathrm{T}}\left(\nabla^{2} L\left(z^{k}, \mu_{k}, \zeta^{k}\right)-B_{k}\right) d_{0}^{k}-(1-\alpha) \eta\left\|F\left(z^{k}, \mu_{k}\right)\right\|+o\left(\left\|d_{0}^{k}\right\|^{2}\right) .
\end{aligned}
$$

Denote $\bar{P}_{*}=E_{n+2 m+1}-A_{*}\left(A_{*}^{\mathrm{T}} A_{*}\right)^{-1} A_{*}^{\mathrm{T}}$, then $\bar{P}_{k} \rightarrow \bar{P}_{*}$. Set

$$
d_{0}^{k}=\bar{P}_{*} d_{0}^{k}+y, \quad y=A_{*}\left(A_{*}^{\mathrm{T}} A_{*}\right)^{-1} A_{*}^{\mathrm{T}} d_{0}^{k} .
$$

Clearly,

$$
\begin{aligned}
y & =A_{*}\left(A_{*}^{\mathrm{T}} A_{*}\right)^{-1}\left(A_{*}-A_{k}\right)^{\mathrm{T}} d_{0}^{k}+A_{*}\left(A_{*}^{\mathrm{T}} A_{*}\right)^{-1} A_{k}^{\mathrm{T}} d_{0}^{k} \\
& =o\left(\left\|d_{0}^{k}\right\|\right)-A_{*}\left(A_{*}^{\mathrm{T}} A_{*}\right)^{-1} F\left(z^{k}, \mu_{k}\right)
\end{aligned}
$$

while, from (2) and (10), it holds that

$$
\|y\|=O\left(\left\|d_{0}^{k}\right\|\right),\|y\|=o\left(\left\|d_{0}^{k}\right\|\right)+O\left(\left\|F\left(z^{k}, \mu_{k}\right)\right\|\right) .
$$

So

$$
\begin{aligned}
\varphi & \leq b\left(\alpha-\frac{1}{2}\right)\left(\left(d_{0}^{k}\right)^{\mathrm{T}} \bar{P}_{*}+y^{\mathrm{T}}\right)\left(\nabla^{2} L\left(z^{k}, \mu_{k}, \zeta^{k}\right)-B_{k}\right) d_{0}^{k}-(1-\alpha) \eta\left\|F\left(z^{k}, \mu_{k}\right)\right\|+o\left(\left\|d_{0}^{k}\right\|^{2}\right) \\
& =b\left(\alpha-\frac{1}{2}\right)\left\|d_{0}^{k}\right\|^{2}+o\left(\left\|d_{0}^{k}\right\|^{2}\right)-(1-\alpha) \eta\left\|F\left(z^{k}, \mu_{k}\right)\right\|+o\left(\left\|F\left(z^{k}, \mu_{k}\right)\right\|\right) \leq 0,
\end{aligned}
$$

which implies the theorem hold.

According to Lemma 4.3, Lemma 4.4 and Lemma 4.5, combining with Theorem 12.3.3 in [15], the following state holds.

Theorem 4.2. The Algorithm B is superlinearly convergent, i.e.,

$$
\left\|\begin{array}{c}
z^{k+1}-z^{*} \\
\mu_{k+1}
\end{array}\right\|=o\left(\left\|z^{k}-z^{*}\right\|\right) .
$$

\section{Conclusion}

By means of perturbed technique and generalized complementarity function, we, using implicit smoothing strategy, equivalently transform the original problem into a family of general optimization problems. Based on the idea of penalty function, the discussed problem is transformed an associated problem with only inequality constraints containing parameter. And then, by providing explicit searching direction, a new variable metric 
gradient projection method for MPCC is established. The smoothing factor $\mu$ regarded as a variable ensures that we can obtain an exact stationary point of original problem once the algorithm terminates in finite iteration. What's more, the proposed algorithm adjusts penalty parameter automatically. Under some mild conditions, the global convergence is obtained as well as the superlinear convergence rate.

\section{Acknowledgements}

The authors are indebted to the anonymous referees for valuable comments and remarks that helped them improve the original version of the paper.

\section{Funding}

This work was supported in part by the National Natural Science Foundation (No. 11361018), the Natural Science Foundation of Guangxi Province (No. 2014GXNSFFA118001), the Key Program for Science and Technology in Henan Education Institution (No. 15B110008) and Huarui College Science Foundation (No. 2014qn35) of China.

\section{References}

[1] Kocvara, M. and Outrata, J. (1994) On Optimization Systems Govern by Implicit Complementarity Problems. Numerical Functional Analysis and Optimization, 15, 869-887. http://dx.doi.org/10.1080/01630569408816597

[2] Fletcher, R., Leyffer, S., Ralph, D. and Scholtes, S. (2006) Local Convergence of SQP Methods for Mathematical Programs with Equilibrium Constraints. SIAM: SIAM Journal on Optimization, 17, 259-286. http://dx.doi.org/10.1137/S1052623402407382

[3] Luo, Z.Q., Pang, J.S. and Ralph, D. (1996) Mathmetical Programs with Equilibrium Constraints. Cambridge University Press, Cambridge. http://dx.doi.org/10.1017/CBO9780511983658

[4] Jiang, H. (2000) Smooth SQP Methods for Mathematical Programs with Nonlinear Complementarity Constaints. SIAM Journal of Optimization, 10, 779-808. http://dx.doi.org/10.1137/S1052623497332329

[5] Kocvara, M. and Outrata, J. (1995) A Nonsmmoth Approach to Optimization Problems with Equilibrium Constraints. In: Ierns, M.C. and Pang, J.D., Eds., Proceedings of the International Conference on Complementatity Problems, SIAM Publications, Baltimore, 148-164.

[6] Outrata, J. (1990) On the Numberical Solution of a Class of Stachelberg Problems. Zeitschrift for Operations Research, 4, 255-278.

[7] Zhang, C., Zhu, Z.B., Chen, F.H. and Fang, M.L. (2010) Sequential System of Linear Equations Algorithm for Optimization with Complementary Constraints. Mathematics Modelling and Applied Computing, 1, 71-80.

[8] Zhang, C., Zhu, Z.B. and Fang, M.L. (2010) A Superlinearly Covergent SSLE Algorithm for Optimization Problems with Linear Complementarity Constraints. Journal of Mathematical Science: Advance and Application, 6, 149-164.

[9] Huang, Z.H., Lin, G.H. and Xiu, N.H. (2014) Several Developments of Variational Inequalities and Complementarity Problems, Bilevel Programming and MPEC. Operations Research Transactions, 18, 113-133.

[10] Zhang, C., Sun, L.M., Zhu, Z.B. and Fang, M.L. (2015) Levenberg-Marquardt Method for Mathematical Programs with Linearly Complementarity Constraints. American Journal of Computational Mathematics, 5, 239-242. http://dx.doi.org/10.4236/ajcm.2015.53020

[11] Outrata, J., Kocvara, M. and Zowe, J. (1998) Nonsmmoth Approach to Aptimization Problems with Equilibrium Constraints. Kluwer Academic Publishers, The Netherland. http://dx.doi.org/10.1007/978-1-4757-2825-5

[12] Gao, Z.Y., He, G.P. and Wu, F. (2004) Sequential Systems of Linear Equations Algorithm for Nonlinear Optimization Problems-General Constrained Problems. Applied Mathematics and Computation, 147, 211-226. http://dx.doi.org/10.1016/S0096-3003(02)00662-8

[13] Jian, J.B., Qin, Y. and Liang, Y.M. (2007) A Generalized Strongly Sub-Feasible Algorithm for Mathematical Problems with Nonliear Complementarity Constraints. Numerical Mathematics: A Journal of Chinese Universities, 29, 15-27.

[14] Zhu, Z.B. and Zhang, K.C. (2004) A New Conjugate Projuction Gradient Method and Superlinear Convergence. Acta Mathematicae Applicatae Sinica, 27, 149-161.

[15] Yuan, Y.X. and Sun, W.Y. (1997) Optimization Theory and Method. Science Press, Beijing. 